\title{
EFECTO DE LA CRISIS FINANCIERA MUNDIAL EN LA CREACIÓN DE VALOR ECONÓMICO DE LOS BANCOS DE LA COMUNIDAD ANDINA EN EL PERIODO 2007-2014
}

\author{
EFFECT OF THE GLOBAL FINANCIAL CRISIS ON THE CREATION OF ECONOMIC \\ VALUE OF THE BANKS OF THE ANDEAN COMMUNITY, 2007-2014
}

OMAR JÁCOME ORTEGA', MARIELLA JÁCOME ORTEGA²

Universidad Católica de Santiago de Guayaquil, Ecuador.xavier.jacome@cu.ucsg.edu.ec

Universidad Católica de Santiago de Guayaquil, Ecuador. mariella.jacome@cu.ucsg.edu.ec

RESUMEN

Esta investigación tiene el objetivo de analizar el efecto de la crisis del sistema financiero mundial con la creación de valor económico de la banca de la Comunidad Andina, durante el período 2007-2014, a partir del método del Valor Económico Ágregado, y el análisis de las variables que caracterizan la generación de valor de los bancos según el referido modelo. Se definió un estudio no experimental, descriptivo, y de tendencia longitudinal. Se tomaron los datos proporcionados por fuentes oficiales de la banca en Bolivia, Colombia, Ecuador y Perú. Se calcularon los indicadores que inciden en la creación de valor económico de cada país, y, según el modelo de Valor Económico Agregado, la posición de cada sistema bancario que conforma la Región Andina. Posterior al año 2008, se evidenció una reducción en el Retorno sobre Patrimonio Inicial medido según el modelo, con una tendencia de menores rendimientos para los años del 2009 en adelante. Se encontró que la crisis impactó en el crecimiento de los activos, provocó una reducción en el rendimiento de los activos productivos y en el rendimiento de la cartera de crédito, constituyéndose estas variables en las más relevantes para el análisis del valor de los bancos de la región.

PALABRAS CLAVE: creación de valor, valor económico agregado, crisis financiera, bancos, comunidad andina.
ABSTRACT

The purpose of this research is to analyze the effect of the crisis in the global financial system on the creation of economic value of the baks of the Andean Community during the period of 2007-2014, based on the Economic Value Added method, and the analysis of the variables that characterize the generation of value of the banks according this model. The study was defined as non-experimental, descriptive, and longitudinal trend. The used data in provided by official sources of banking from Bolivia, Colombia, Ecuador and Peru. The indicators that affect the creation of economic value of each country were calculated, and, according to the Economic Value Added model, the position of each banking system of the Andean Region. According to the model, after 2008, there was a reduction in the Return on Initial Equity measured, with a trend of lower yields for the years of 2009 and onwards. It was found that the crisis impacted the growth of assets, caused a reduction in the yield of productive assets and in the performance of the credit portfolio, these variables were the most relevant for the analysis of the value of the banks of the region.

KEYWORDS: creation of value, economic value added, financial crisis, banks, andean community. 
INTRODUCCIÓN

Entre los años 2003 y 2007, América Latina experimentó el crecimiento económico más acelerado. El auge fue amplio y más sólido en las economías pequeñas y medianas que en las dos economías más grandes de la región, como lo son Brasil y México (Ocampo, 2011).

Esta coyuntura favorable del período 20032007 para América Latina estuvo basada en una combinación inusual de auge financiero, bonanza excepcional de precios de los productos básicos y nivel elevado de remesas de los trabajadores migrantes (Ocampo, 2009).

Desde el 2001 al 2007 la política monetaria de los Estados Unidos fue volátil. En 2001, la tasa de interés pasó del $6 \%$ al 1,75\%, continuó la reducción en 2003 hasta el 1\% para promover el crecimiento; en 2005-2006, elevó la tasa hasta el $5,25 \%$, con el objetivo de reducir la inflación aunque sin éxito, y sin considerar sus efectos sobre prestatarios y productores; en 2007-2008, volvió a reducirse hasta el 1\% para evitar la recesión, sin embargo, esta se produjo de forma inevitable (Ferrari, 2008).

Adicional al deficiente manejo de la política monetaria señalado, existieron eventos que impulsaron la crisis hipotecaria subprime (alto riesgo) y reventaron la burbuja inmobiliaria, los cuales Zurita González, Martínez Pérez, y Rodríguez Montoya (2009); y Serrano Rodríguez (2009), resumen en:

- Desaceleración de la economía de Estados Unidos.

- Oferta de viviendas nuevas y usadas comenzó a superar la demanda, por lo que los precios de las comenzaron a reducirse.

- Gran incertidumbre en los mercados.

- Deficiente regulación y falta de independencia en las calificadoras de riesgo.

Esta crisis tuvo su hecho más crítico con la declaración de bancarrota de Lehman Brothers, en septiembre de 2008, marcando al segundo semestre de este año como el periodo en que se desencadenó la más grave crisis financiera y económica desde la Gran Depresión en los años 30.

La crisis financiera global produjo una profunda recesión en América Latina en el último trimestre de 2008 y el primero de 2009. El impacto inicial sobre el Producto Interno Bruto (PIB) fue más fuerte que en los países de la Organización para la Cooperación y el Desarrollo Económico (OCDE) en términos absolutos y, en especial, con relación a las tasas de crecimiento imperantes durante los años del auge (Ocampo, 2011).

Las formas de contagio de la crisis en América Latina se dieron por la reducción del precio de materias primas, explicado por una menor demanda agregada; reducción de las remesas de los inmigrantes a los países en desarrollo; y cambios en los flujos de capitales e inversión extranjera directa basados en una preferencia de inversiones más líquidas y seguras, en lugar de aquellas más rentables (Ferrari, 2008).

Un aspecto importante de los países que conforman la Comunidad Andina (Colombia, Bolivia, Ecuador y Perú), además de la intensiva migración entre ellos, es la fuerte migración internacional que se manifiesta en una significativa presencia de ciudadanos andinos que viven en Estados Unidos, Canadá y, en especial, Europa. El impacto de las remesas tiende a ser mayor en los países pequeños (como es el caso de Ecuador), con altos niveles de pobreza y con una estructura productiva menos diversificada (Neira Orjuela, 2009).

Las consecuencias económicas provocadas a raíz de la crisis financiera mundial del 2008 demandan un análisis de la situación del sistema bancario de las economías más próximas y de constante comparación en términos de competitividad, como son los miembros de la Comunidad Andina. Las políticas económicas adoptadas por cada uno de estos países, las condiciones en que se desempeñan sus agentes económicos y las características intrínsecas de cada país, pueden haber creado factores que diferencian la posición financiera y la solvencia de sus sistemas bancarios.

Posterior al 2008, las consecuencias sociales y económicas han sido dramáticas, y se manifiestan en despidos masivos, cierres de empresas y caída en la actividad económica de varios países. En el 2009, la economía mundial se redujo por primera vez después de la segunda guerra mundial, con porcentajes de decrecimiento en las economías desarrolladas.

Los países andinos experimentaron una desaceleración en el crecimiento anual del PIB en el 2009, recuperándose en los años siguientes. Las principales economías de la Comunidad Andina se ubicaron en torno al 1\% en ese período, como es el caso de Colombia con un crecimiento del $1.65 \%$ y Perú de $1.05 \%$, lo que se puede observar en la tabla 1.

Los activos bancarios de la Comunidad Andina han tenido oscilaciones en el período 2007-2014, lo cual está relacionado con el cre- 
TABLA 1. CRECIMIENTO DE PIB (\% ANUAL)

\begin{tabular}{lccccccccr|} 
PAís & $\mathbf{2 0 0 7}$ & $\mathbf{2 0 0 8}$ & $\mathbf{2 0 0 9}$ & $\mathbf{2 0 1 0}$ & $\mathbf{2 0 1 1}$ & $\mathbf{2 0 1 2}$ & $\mathbf{2 0 1 3}$ & $\mathbf{2 0 1 4}$ & PROMEDI0 \\
\hline Bolivia & 4.56 & 6.15 & 3.36 & 4.13 & 5.17 & 5.18 & 6.78 & 5.40 & 5.09 \\
\hline Colombia & 6.90 & 3.55 & 1.65 & 3.97 & 6.59 & 4.04 & 4.94 & 4.55 & 4.52 \\
\hline Ecuador & 2.19 & 6.36 & 0.57 & 3.53 & 7.87 & 5.22 & 4.64 & 3.80 & 4.27 \\
\hline Perú & 8.52 & 9.14 & 1.05 & 8.45 & 6.45 & 5.95 & 5.77 & 2.35 & 5.96 \\
\hline
\end{tabular}

Fuente: Banco Mundial (2015)

cimiento de la economía de los países de la región. El crecimiento de los activos de los bancos andinos del 2008, en comparación al 2007, fue de $29 \%$, no obstante la variación del 2009 res- $^{-}$ pecto al año anterior es próxima a cero. En los años siguientes se evidencia una recuperación en el crecimiento de los activos de los bancos de la región, cuyos datos se presentan en la tabla 2.

TABLA 2. CRECIMIENTO DE LOS ACTIVOS BANCARIOS (\% ANUAL)

\begin{tabular}{lrrrrrrr|} 
REGIÓN & $\mathbf{2 0 0 8}$ & $\mathbf{2 0 0 9}$ & $\mathbf{2 0 1 0}$ & $\mathbf{2 0 1 1}$ & $\mathbf{2 0 1 2}$ & $\mathbf{2 0 1 3}$ & $\mathbf{2 0 1 4}$ \\
\hline CAN & 29.0 & -0.3 & 31.1 & 21.2 & 18.5 & 11.6 & 7.7 \\
\hline
\end{tabular}

Fuente: Organismos de supervisión bancaria de los países de CAN

Ante estos hechos económicos, es necesario identificar los posibles factores que ocasionaron esta crisis sistémica. El Fondo Monetario Internacional (2014) resume las causas de la crisis financiera mundial en la debilidad de las instituciones financieras, la regulación y supervisión inadecuadas y la falta de transparencia en la gestión. En su informe sobre la Solidez del Sistema Financiero resalta que la crisis reciente destacó la importancia de una supervisión y gestión eficaz del riesgo sistémico.

La ausencia de controles, supervisión y regulación a las entidades financieras es la principal causa de la crisis financiera-económica internacional. Las políticas de control y los instrumentos fallaron, y los organismos de supervisión no ejercieron su rol en el sistema. Por tanto, se destacan como problemas en esta crisis, la ausencia de métodos que midan la estabilidad y solvencia del sistema financiero de los países y la falta de ética en el manejo de la información por parte de los gestores de las entidades financieras y de los supervisores del sistema.

Seis años después de la explosión de la crisis financiera, el Fondo Monetario Internacional (2014) señala que el sistema financiero mundial está experimentando una serie de transiciones difíciles en la senda hacia una mayor estabilidad. Las economías de mercados emergentes se encuentran en transición hacia un crecimiento más sostenible del sector financiero, al tiempo que abordan vulnerabilidades macroeconómicas en un ambiente financiero externo menos favorable.
En la evaluación reciente de la economía de América Latina y el Caribe, el Banco Mundial (2014) señala que la desaceleración en China y la baja prolongada de los precios de los productos básicos afectará las exportaciones de los países de la región, los ingresos por concepto de exportaciones y también las inversiones para mantener el crecimiento cercano a su potencial. En general, con las brechas de producción mayormente cerradas y la disminución de los excedentes de capacidad, hay poco campo para acelerar de manera sostenida el crecimiento sin generar desequilibrios macroeconómicos

Es importante el abordaje de esta temática puesto que los problemas en los sistemas financieros no solo perturban la intermediación financiera, sino que también pueden socavar la eficacia de la política monetaria, exacerbar las desaceleraciones de la economía, desencadenar la fuga de capitales y presiones cambiarias y crear un elevado costo fiscal si hiciera falta sanear las instituciones financieras que atraviesan dificultades. Además, dada la creciente conectividad entre las instituciones financieras y los vínculos financieros y comerciales cada vez más estrechos entre los países, los shocks financieros de una jurisdicción pueden extenderse rápidamente entre sectores financieros y más allá de las fronteras nacionales (Fondo Monetario Internacional, 2014).

Considerando los antecedentes históricos de la crisis financiera global y la condición actual de la economía mundial, se necesita evaluar la capacidad de creación de valor económico de la banca de los países de la Región Andina ante un escenario de liquidez reducida y con presiones fiscales, por lo que se puede resumir el problema planteado en esta investigación a través de la siguiente pregunta: ¿Cómo afectó la crisis del sistema financiero mundial en la creación de valor económico de los bancos de la Comunidad Andina durante el período 2007-2014?

Para abordar este problema se planteó como objetivo general de investigación: analizar el efecto de la crisis del sistema financiero mundial del año 2008 en la creación de valor económico de los bancos de la Comunidad Andina, realizando una aplicación del modelo de Valor 
Económico Agregado (EVA) y su relación con las variables que impulsan el valor.

\section{METODOLOGÍA}

El propósito de esta investigación se alcanza a partir de la definición de una perspectiva teórica del modelo, aplicado a través de un estudio no experimental, considerando los siguientes aspectos metodológicos definidos por Hernández, Fernández-Collado, y Baptista (2007):

- Proceso formal: método hipotético-deductivo

- Grado de abstracción: investigación aplicada

- Metodología: cuantitativa

- Naturaleza de los objetivos: investigación descriptiva

- Diseño de investigación: longitudinal de tendencia.

MUESTRA

Este estudio analizó los datos del sistema bancario, comprendidido por los bancos de cada país de la Comunidad Andina, período 2007-2014, no se consideraron las entidades financieras estatales.La población está compuesta por los sistemas bancarios de los países de la Comunidad Andina.

DATOS

Fueron obtenidos de una fuente secundaria de información, tomados a partir de las bases de datos oficiales de los organismos de control en cada país:

- Ecuador: Superintendencia de Bancos y Seguros

- Colombia: Superintendencia Financiera

- Perú: Superintendencia de Banca, Seguros y AFP

- Bolivia: Autoridad de Supervisión del Sistema Financiero

MÉTODOS Y TÉCNICAS DE CÁLCULO DEL EVA

El EVA es una medida operativa de creación de valor desarrollada por Stern Stewart, que ha tenido la finalidad de ser una medida explicativa del valor, una guía para la gestión interna y toma de decisiones, y un método para el establecimiento de los planes de retribución para los directivos de las empresas. De acuerdo a los trabajos realizados por Biddle, Bowen y Wallace (1997), Iñiguez y Poveda (2001), Worthington y West (2001) y Venanzi (2010) el concepto que sustenta el EVA se remonta a finales del siglo XVIII, cuando sesostenía que para que una compañía creara valor era necesario obtener una rentabilidad superior al coste de los recursos empleados. Posteriormente, el tema ha sido abordado bajo diversos términos, hasta que en la década de los noventa, el planteamiento del EVA alcanzó una gran difusión y aplicación en empresas de gran relevancia.

Según Stewart (1991) y Stern, Shiely y Ross (2002), el Valor Económico Agregado (EVA) es la medida interna de desempeño operacional que mejor refleja el éxito de las compañías en agregar valor a sus accionistas. Stewart planteó conceptualmente el cálculo del EVA de la siguiente forma:

EVA $=$ (Tasa de Retorno - Costo de Patrimonio o Capital) $x$ Patrimonio.

Es decir, que el EVA es igual a la diferencia de la Tasa de Rendimiento y el Costo de Capital, multiplicada por el Capital Invertido.

En resumen, el EVA podría definirse como el monto resultante, deducidos de los ingresos el total de los gastos, incluidos el costo de oportunidad del capital y los impuestos. Esta métrica considera la productividad de todos los factores utilizados para desarrollar la actividad empresarial (Amat, 1999).

La fórmula del EVA ajustada para el cálculo como medida de desempeño de la banca es (Fraker, 2006):

EVA $=$ NOPAT - Patrimonio $x \%$ Costo del Patrimonio

- NOPAT: Net operating profit after taxes o Utilidades ordinarias netas después de impuestos.

- Patrimonio: Corresponde al patrimonio invertido en la institución bancaria.

- Costo del Patrimonio: Corresponde al costo de capital o patrimonio invertido en el banco.

El resultado de esta fórmula se interpreta en el sentido de que si produce un valor positivo, el mismo representa un incremento del valor de los accionistas, y uno negativo se interpreta como una disminución del valor.

El cálculo del NOPAT (Utilidades ordinarias netas después de impuestos) comprende la depuración de las utilidades ordinarias netas después de impuestos, extrayendo de esta magnitud aquellas cifras extraordinarias o que son calculadas de acuerdo a principios contables, lo cual a menudo distorsiona la situación real 
económica de la firma. Los ejemplos característicos son las depreciaciones de activo fijo, las amortizaciones o provisiones contabilizadas en la cuenta de resultados.

Una diferencia sustancial entre los bancos y los otros tipos de empresas es el rol de la deuda, para las instituciones no financieras la deuda forma parte de las operaciones financieras y por lo tanto los gastos e ingresos financieros son excluidos del cálculo del NOPAT. En el caso de un banco, los fondos de la deuda son la materia prima, la cual es intermediada para ser transformada en activos generadores de ingresos, como los créditos. Bajo esta perspectiva, para un banco los intereses causados por la deuda son equivalentes al costo de los bienes vendidos (Popa, Mihailescu, \& Caragea, 2009).

Considerando este criterio, los intereses causados o gastos financieros producidos por la actividad de intermediación son incluidos en el cálculo del NOPAT, como parte de las utilidades ordinarias.

Adicionalmente a los ajustes que se realizan a las empresas no financieras, existen otros comunes para un banco, los cuales son la exclusión de las provisiones realizadas por pérdidas bancarias, las provisiones por impuestos, y valores correspondientes a cuentas no recurrentes, ganancias no realizadas o provisiones por pérdidas en negociación de valores (Popa, Mihailescu, \& Caragea, 2009).

La determinación del Capital invertido, siguiendo la literatura sobre el EVA muestra varias acepciones (Costa, 2012):

1. Considerar el total invertido por los accionistas y pasivos financieros, sin embargo, esta metodología no es aplicable a instituciones financieras dado que los pasivos financieros de los bancos no cumplen la misma función que las fuentes de financiación en las compañías no financieras.

2. Enfocarse en los activos, considerando el capital invertido como la diferencia entre el valor de mercado de los activos y los pasivos no financieros.

En un sentido más práctico, la determinación del capital invertido se calcula tomando en cuenta el patrimonio total del banco, que está resumido en la suma de las categorías Tier 1 y Tier 2 de patrimonio.

No se consideran los fondos pertenecientes a la deuda y los depósitos del público dado que estos corresponden a la mecánica operacional del negocio de intermediación financiera, cuyo impacto es incluido en el resultado operacional de la entidad bancaria.

El cálculo del costo del capital se refiere al rendimiento exigido por los accionistas en relación al monto invertido en la institución bancaria, y es igual a:

Costo del Capital propio o Patrimonio $=$ Patri $^{-}$ monio $\mathrm{x} \%$ Retorno requerido

El porcentaje de retorno sobre el patrimonio que demandan los accionistas de un banco está basado en el hecho de que ellos requieren, por una parte, un retorno por los fondos invertidos en el negocio, y por otra, un retorno que refleje el riesgo inherente de invertir en una entidad bancaria.

El modelo de valuación de activos de capital (CAPM, del inglés Capital Asset Pricing Model) nos permite calcular el porcentaje del costo del capital o retorno requerido (Allen, Myers, \& Brealey, 2010). La fórmula básica es la siguiente:

Costo del capital o patrimonio \% = Tasa libre de riesgo + (Coef. Beta x Prima de Riesgo de Mercado) + Prima de Riesgo País

- Tasa libre de riesgo: Es la tasa de interés ganado en una inversión que no supone riesgo, comúnmente hace referencia al interés pagado por los bonos emitidos por el Tesoro de Estados Unidos o en el caso de Europa los bonos alemanes.

- Coeficiente Beta: Es el nivel de riesgo de la inversión en una empresa de un sector específico (en esta investigación, hace referencia al sector bancario) en relación al mercado general de acciones.

- Prima de Riesgo de mercado: Es el riesgo asociado con la inversión en el mercado accionario como un todo.

- Riesgo País: Es el riesgo implícito asociado a la posición de un país según variables económicas, políticas y financieras.

\section{SISTEMA DE VARIABLES}

Los aspectos principales en que se centra el análisis de un banco son las fuentes, calidad y sostenibilidad de las ganancias, la liquidez y la suficiencia de capital (Barltrop \& McNaughton, 1992). En este mismo sentido Fernández (2013) señala que el análisis del valor del patrimonio depende de tres factores principales (Variables impulsoras de valor): las expectativas de los 
flujos futuros, retorno requerido del patrimonio y comunicación con el mercado, lo cual se pone de manifiesto en los estudios de Fernández y Bermejo (2008); y Fernández, Aguirreamalloa, y Corres (2011).

López Lubián y Vilela (2000) señalan que las entidades bancarias tienen características diferenciadoras que provocan que el proceso de creación de valor sea diferente al de otros negocios. La revisión de trabajos de diversos autores nos permite encontrar coincidencias y establecer nuevos aspectos a ser considerados en la definición de las variables creadoras de valor para un banco, las cuales resumimos a partir del trabajo de Wittrup, y Jensen (2012):

Según Koller, Goedhart, y Wessels (2010), las principales variables que definen la creación de valor son: las tasas de interés de ingresos y costos; el valor en libros de los activos, pasivos y patrimonio; la relación de Gastos dividido para Ingresos de intereses netos; la relación de capital o patrimonio según los activos; el Costo del patrimonio; el crecimiento; y las provisiones de las pérdidas por préstamos.

Fiordelisi y Molyneux (2010) resaltan que las tres principales variables impulsoras de valor para el caso de los bancos son:

a. Ganancia Operativa neta.

b. Coste de oportunidad del capital.

c. Capital invertido.

Gross (2006) en su trabajo empírico sobre 139 bancos alemanes en el período 1998-2003, busca encontrar las métricas que son capaces de cuantificar y comprender los factores fundamentales del valor. Se considera como variable dependiente el Ingreso residual, que es explicado por indicadores financieros y por impulsores operacionales de valor. Los hallazgos de este estudio sugieren que sólo la rentabilidad y el riesgo son controladores relevantes para el valor del accionista en los bancos.

Según lo planteado por los autores señalados anteriormente, se definió en este estudio que las variables relacionadas a la creación de valor económico que deben ser estudiadas para el caso de los bancos de la Comunidad Andina son: el crecimiento del volumen, la mezcla de los activos productivos e improductivos, la mezcla de activos en riesgo, la mezcla de fuentes con costo, el margen financiero, y el costo de capital.

La tabla 3 define conceptual y operacionalmente los indicadores de creación de valor económico para el caso de la banca según el método de Valor Económico Agregado (EVA) tomando como referencia el trabajo doctoral de Jácome Ortega, X.O. (2015).

\section{RESULTADOS}

En primera instancia la presentación de los resultados de la investigación abarca el análisis de los indicadores que se relacionan con la creación de valor económico, como son: el crecimiento del volumen o activos, la mezcla de activos productivos, la mezcla de activos de riesgo (cartera), la mezcla de fuentes con costo, el margen financiero, y el costo de capital.

Posterior a la revisión de los resultados de los indicadores, se realiza el cálculo del EVA para cada sistema bancario de la Comunidad Andina. Se presentan las tablas de cálculo del EVA de cada país y su evolución en el período 2007-2014.

TABLA 3. INDICADORES DE CREACIÓN DE VALOR Y DEFINICIONES

\begin{tabular}{|c|c|c|}
\hline INDICADOR & DEFINICIÓN CONCEPTUAL & DEFINICIÓN OPERACIONAL \\
\hline Crecimiento del volumen & Aumento o disminución del nivel de activos del banco. & $\begin{array}{l}\text { Representa la variación absoluta y relativa en la cifra de Activos de un } \\
\text { período respecto al inmediato anterior. }\end{array}$ \\
\hline $\begin{array}{l}\text { Mezcla activos } \\
\text { productivos e } \\
\text { improductivos }\end{array}$ & $\begin{array}{l}\text { Relación existente entre activos que tienen capacidad } \\
\text { producir ingresos financieros y los que no tienen. }\end{array}$ & $\begin{array}{l}\text { Es la composición porcentual de los activos según su incidencia en la } \\
\text { generación de ingresos. }\end{array}$ \\
\hline $\begin{array}{l}\text { Mezcla de activos en } \\
\text { riesgo }\end{array}$ & $\begin{array}{l}\text { Se refiere a la composición de los activos según su } \\
\text { nivel de riesgo. }\end{array}$ & $\begin{array}{l}\text { Considera la composición del portafolio de inversiones y cartera de una } \\
\text { entidad bancaria. }\end{array}$ \\
\hline $\begin{array}{l}\text { Mezcla de fuentes con } \\
\text { costo }\end{array}$ & $\begin{array}{l}\text { Clasificación de las fuentes de pasivos según su costo } \\
\text { financiero. }\end{array}$ & $\begin{array}{l}\text { Composición porcentual de los tipos de pasivos según su costo. Los pasivos } \\
\text { son los depósitos de terceros (a la vista } 0 \text { a plazo) y las obligaciones. }\end{array}$ \\
\hline Margen financiero & $\begin{array}{l}\text { El margen financiero es la utilidad obtenida sobre las } \\
\text { operaciones financieras. }\end{array}$ & $\begin{array}{l}\text { Diferencia entre ingresos financieros (intereses y rendimientos) y costo de los } \\
\text { pasivos (intereses, rendimientos, provisiones). }\end{array}$ \\
\hline Costo del capital & Representa el rendimiento exigido por los accionistas. & $\begin{array}{l}\text { Representa el costo de los recursos propios, calculados a partir del Modelo } \\
\text { de Valuación de Activos de Capital. }\end{array}$ \\
\hline EVA & $\begin{array}{l}\text { Medida de creación valor desarrollada por Stern } \\
\text { Stewart al obtener rentabilidad superior al costo } \\
\text { recursos invertidos. }\end{array}$ & $\begin{array}{l}\text { Es igual al NOPAT (beneficio neto después de impuestos, antes de partidas } \\
\text { extraordinarias y gastos de intereses por financiación ajena) menos el costo } \\
\text { de capital invertido. }\end{array}$ \\
\hline
\end{tabular}


CRECIMIENTO DEL VOLUMEN (TOTAL DE ACTIVOS)

El análisis del crecimiento del volumen del sistema bancario de los países de la Región Andina nos indica que el promedio de la región es del $17 \%$, donde se resalta el crecimiento de Bolivia y Perú, con un $21 \%$ y $17.2 \%$, respectivamente. El crecimiento de Colombia está próximo al de la región, aunque ligeramente inferior con $16.28 \%$. El resultado de Ecuador en este indicador está en $13.64 \%$, distante del promedio regional por tres y medio puntos porcentuales. Esto se puede observar en la tabla 4.

TABLA 4. CRECIMIENTO DE ACTIVOS (VOLUMEN)

\begin{tabular}{lrrrr}
\hline AÑO & BOLIVIA & COLOMBIA & ECUADOR & PERÚ \\
\hline 2008 & $30.49 \%$ & $23.14 \%$ & $19.51 \%$ & $46.11 \%$ \\
\hline 2009 & $18.62 \%$ & $-0.98 \%$ & $6.77 \%$ & $-4.01 \%$ \\
\hline 2010 & $10.21 \%$ & $36.38 \%$ & $17.52 \%$ & $29.31 \%$ \\
2011 & $21.10 \%$ & $25.25 \%$ & $15.88 \%$ & $14.41 \%$ \\
\hline 2012 & $19.56 \%$ & $17.23 \%$ & $16.80 \%$ & $22.03 \%$ \\
2013 & $17.23 \%$ & $10.78 \%$ & $10.27 \%$ & $13.14 \%$ \\
2014 & $33.45 \%$ & $6.27 \%$ & $9.37 \%$ & $6.02 \%$ \\
\hline PROMEDIO & $\mathbf{2 1 . 5 2 \%}$ & $\mathbf{1 6 . 8 7 \%}$ & $\mathbf{1 3 . 9 5 \%}$ & $\mathbf{1 8 . 1 5 \%}$ \\
\hline
\end{tabular}

El análisis de las tendencias de crecimiento de los activos bancarios de los países de la Comunidad Andina, mostrado en el gráfico 1 , nos permite resaltar el impacto de la crisis financiera en el año 2009 con la presencia de una desaceleración marcada, e incluso con decrecimientos en las principales economías de la región, como son $\mathrm{Co}^{-}$ lombia y Perú. En los años siguientes se observa una recuperación del crecimiento del volumen de los bancos, principalmente del 2010 al 2012. En los años 2013 y 2014 se presenta una tendencia decreciente excepto para Bolivia, que tiene un repunte hacia el final del período analizado.

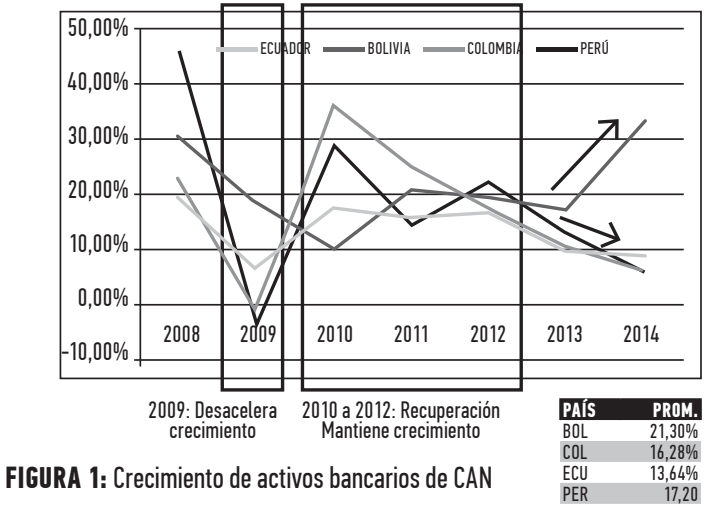

\section{ACTIVOS PRODUCTIVOS, MEZCLA DE CARTERA Y MEZCLA DE FUENTES CON COSTO}

La calidad de activos se mide por la proporción del total de activos que son productivos, es decir, que tienen capacidad de generar ingresos financieros. El promedio de la Región Andina del período $2007-2014$ es $85.68 \%$.

La mezcla de activos en riesgo se mide por la proporción que representa la cartera de créditos en los activos productivos. El promedio de la región para el período de estudio es $68.60 \%$.

La mezcla de fuentes con costo representa el porcentaje de la suma del pasivo y patrimonio que genera pago por concepto de intereses y/o comisiones. El resultado de este indicador para la región es $71.82 \%$ promedio entre el 2007 al 2014. Esto se puede observar en la tabla 5.

TABLA 5. PROMEDIO DE CALIDAD DE ACTIVOS, MEZCLA DE CARTERA Y MEZCLA DE FUENTES CON COSTO PERÍODO DEL 2007 AL 2014

\begin{tabular}{lccccc} 
INDICADOR & BOLIVIA & COLOMBIA & ECUADOR & PERÚ & PROMEDIO \\
$\begin{array}{l}\text { Mezcla de } \\
\begin{array}{l}\text { activos } \\
\text { productivos }\end{array}\end{array}$ & $79.68 \%$ & $91.25 \%$ & $86.63 \%$ & $85.14 \%$ & $85.68 \%$ \\
$\begin{array}{l}\text { Mezcla de } \\
\text { cartera }\end{array}$ & $69.97 \%$ & $70.28 \%$ & $63.44 \%$ & $70.71 \%$ & $68.60 \%$ \\
$\begin{array}{l}\text { Mezcla de } \\
\text { fuentes con } \\
\text { costo }\end{array}$ & $57.14 \%$ & $82.76 \%$ & $60.64 \%$ & $86.74 \%$ & $71.82 \%$ \\
\hline
\end{tabular}

Bolivia tiene un nivel de activos productivos y proporción de fuentes con costo inferior al promedio regional. La mezcla de cartera está próxima al promedio.

Colombia tiene el nivel más alto de proporción de activos productivos de la región, y un alto nivel de mezcla de fuentes con costo. El nivel de mezcla de cartera es superior al promedio regional.

Ecuador tiene un nivel superior de proporción de activos productivos, el nivel más bajo de mezcla de cartera de la región, y el segundo más bajo nivel de mezcla de fuentes con costo. La posición de mezcla de cartera y mezcla de fuentes con costo están equilibradas.

Perú presenta un nivel de proporción de activos productivos próximo al promedio regional, un nivel superior en mezcla de cartera, y el nivel más alto en la proporción de fuentes con costo.

MARGEN FINANCIERO

El Margen Financiero es igual a la diferencia del Rendimiento de los Activos Productivos y el Rendimiento de las Fuentes con Costo. El Rendimiento de los Activos Productivos es igual a la suma del Rendimiento de la Cartera multiplicado por la Mezcla de Cartera y el Rendimiento de las Inversiones multiplicado por la Mezcla de las Inversiones. 
En el período de estudio, el promedio regional del Margen Financiero es de 5.74\% resultante de una diferencia de $8.83 \%$ de Rendimiento promedio de Activos Productivos y $3.10 \%$ de Rendimiento promedio de Fuentes con Costo.

El promedio de la Región Andina en Rendimiento de la Cartera es $11.05 \%$ y $4.15 \%$ en Rendimiento de Inversiones.

El nivel promedio más alto de Margen Financiero entre los años analizados lo tiene la banca de Perú con $7.55 \%$, sustentado en el mayor nivel de Rendimiento de Activos Productos de 10.17\% y en el más bajo nivel de Rendimiento de Fuentes con Costo de $2.65 \%$. El nivel más bajo es el de Ecuador, con $4.96 \%$, explicado por el nivel más bajo de Rendimiento de Activos Productivos de $8.04 \%$, que no es compensado por el Rendimiento de las Fuentes con Costo, ubicándose este porcentaje próximo al promedio. Esto se puede observar en la tabla 6.

TABLA 6. PROMEDIO DE RENDIMIENTOS Y MARGEN FINANCIERO DE REGIÓN ANDINA PARA EL PERÍODO 2007-2014

\begin{tabular}{lccccc}
\hline INDICADOR & BOLIVIA & COLOMBIA & ECUADOR & PERÍ & PROMEDIO \\
\hline $\begin{array}{l}\text { (+) Rendimiento } \\
\text { Activos }\end{array}$ & $8.20 \%$ & $8.91 \%$ & $8.04 \%$ & $10.17 \%$ & $8.83 \%$ \\
$\begin{array}{l}\text { Productivos } \\
\quad \text { Rend. Cartera }\end{array}$ & $10.20 \%$ & $11.20 \%$ & $11.46 \%$ & $11.33 \%$ & $11.05 \%$ \\
$\begin{array}{l}\text { Rend. } \\
\text { Inversiones }\end{array}$ & $3.41 \%$ & $3.58 \%$ & $2.15 \%$ & $7.48 \%$ & $4.15 \%$ \\
$\begin{array}{l}\text { (-) Rendimiento } \\
\text { Fuentes con } \\
\text { Costo }\end{array}$ & $2.85 \%$ & $3.79 \%$ & $3.09 \%$ & $2.65 \%$ & $3.10 \%$ \\
\hline $\begin{array}{l}\text { (=) Margen } \\
\text { Financiero }\end{array}$ & $5.31 \%$ & $5.15 \%$ & $4.96 \%$ & $7.55 \%$ & $5.74 \%$ \\
\hline
\end{tabular}

COSTO DE CAPITAL

El costo de capital es una variable relevante en el proceso de creación de Valor Económico. Se tomó como fuente los datos calculados por Damodaran (2014) para cada país como el Riesgo asociado del Patrimonio. El nivel de costo porcentual se presenta para cada año de cálculo reflejando la variabilidad de riesgo de cada economía de la Comunidad Andina en el período analizado. Esto se puede observar en la tabla 7.

\section{CREACIÓN DE VALOR ECONÓMICO AGREGADO}

La crisis financiera mundial del 2008 impactó en la capacidad de creación de valor económico de la banca de la Comunidad Andina. Esta aseveración guarda relación con el impacto en el crecimiento de los activos bancarios. Adicionalmente, se puede observar la reducción del Retorno sobre el patrimonio inicial posterior al año 2008 en la banca de todos los países.

A continuación se presentan los cálculos del EVA para cada país, presentando de forma desagregada el cálculo del NOPAT, el Retorno sobre Patrimonio que se calcula dividiendo el NOPAT para el Patrimonio inicial, el Margen que se calcula restando al Retorno el Costo de capital, y el EVA que es igual a la multiplicación del Margen por el Patrimonio Inicial. Los datos se presentan en dólares para todos los países.

En el caso de Bolivia, el Retorno sobre el patrimonio inicial estaba en su mejor nivel al fin del 2008, resultando un $63 \%$. Posterior a ese año, el indicador refleja una reducción, con un promedio en torno al 50\%, como se presenta en la tabla 8 .

TABLA 7. COSTO DE CAPITAL DE REGIÓN ANDINA PARA EL PERÍODO 2007-2014

\begin{tabular}{lrrrrrrrr|} 
AÑoS & $\mathbf{2 0 0 7}$ & $\mathbf{2 0 0 8}$ & $\mathbf{2 0 0 9}$ & $\mathbf{2 0 1 0}$ & $\mathbf{2 0 1 1}$ & $\mathbf{2 0 1 2}$ & $\mathbf{2 0 1 3}$ & $\mathbf{2 0 1 4}$ \\
\hline Bolivia & $6,82 \%$ & $18,50 \%$ & $12,75 \%$ & $11,00 \%$ & $12,00 \%$ & $10,68 \%$ & $10,40 \%$ & $11,15 \%$ \\
\hline Colombia & $6,82 \%$ & $8,90 \%$ & $7,50 \%$ & $8,00 \%$ & $9,00 \%$ & $8,80 \%$ & $8,30 \%$ & $8,60 \%$ \\
\hline Ecuador & $11,54 \%$ & $18,50 \%$ & $19,50 \%$ & $20,00 \%$ & $18,75 \%$ & $16,30 \%$ & $16,25 \%$ & $15,50 \%$ \\
\hline Perú & $6,82 \%$ & $8,90 \%$ & $7,50 \%$ & $8,00 \%$ & $9,00 \%$ & $8,43 \%$ & $7,85 \%$ & $7,55 \%$ \\
\hline
\end{tabular}

TABLA 8. CÁLCULO DEL EVA DE BANCA DE BOLIVIA 2007-2014

\begin{tabular}{|c|c|c|c|c|c|c|c|c|c|}
\hline is & AÑos & 2007 & 2008 & 2009 & 2010 & 2011 & 2012 & 2013 & 2014 \\
\hline$\dot{=}$ & Tipo de Cambio & 7.574 & 7.054 & 7.057 & 6.973 & 6.904 & 6.944 & 6.945 & 6.931 \\
\hline ヘิ & Resultado del Ejercicio & 104,251 & 123,289 & 142,723 & 130,947 & 168,620 & 183,733 & 173,060 & 269,432 \\
\hline $\bar{\sigma}_{j}$ & Provisiones & 87,207 & 184,346 & 170,539 & 255,772 & 211,192 & 230,441 & 270,912 & 401,940 \\
\hline$\dot{\infty}$ & Depreciaciones y Amortizaciones & 16,107 & 19,219 & 22,263 & 26,162 & 29,815 & 36,747 & 40,944 & 56,846 \\
\hline ஜ் & NOPAT & 207,565 & 326,854 & 335,524 & 412,881 & 409,627 & 450,921 & 484,915 & 728,218 \\
\hline 요 & Patrimonio Inicial & 442,582 & 519,142 & 658,508 & 728,301 & 778,826 & 942,759 & $1,154,696$ & $1,281,706$ \\
\hline 㒸市 & Retorno sobre Patrimonio & $46.90 \%$ & $62.96 \%$ & $50.95 \%$ & $56.69 \%$ & $52.60 \%$ & $47.83 \%$ & $42.00 \%$ & $56.82 \%$ \\
\hline 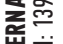 & Costo de Capital & $6.82 \%$ & $18.50 \%$ & $12.75 \%$ & $11.00 \%$ & $12.00 \%$ & $10.68 \%$ & $10.40 \%$ & $11.15 \%$ \\
\hline 崫忌 & MARGEN (Retorno - Costo) & $40.08 \%$ & $44.46 \%$ & $38.20 \%$ & $45.69 \%$ & $40.60 \%$ & $37.15 \%$ & $31.60 \%$ & $45.67 \%$ \\
\hline 12 & EVA & 177,381 & 230,813 & 251,565 & 332,768 & 316,168 & 350,234 & 364,827 & 585,308 \\
\hline
\end{tabular}


En el caso de Colombia, el indicador Retorno sobre Patrimonio inicial estaba alrededor del $70 \%$ en los años 2007 y 2008, posterior a esos años se observa una reducción significativa en los años siguientes (2009-2014) con resultados próximos al $50 \%$. Los datos se presentan en la tabla 9.
La crisis financiera mundial impactó en el Retorno medido según el método EVA, calculado a partir de la relación del NOPAT sobre el Patrimonio inicial. La figura 2 nos muestra la evolución del indicador de Retorno según EVA, destacando que al inicio del período analizado

TABLA 9. CÁLCULO DEL EVA DE BANCA DE COLOMBIA 2007-2014

\begin{tabular}{lrrrrrrrr} 
AÑ̃S & $\mathbf{2 0 0 7}$ & $\mathbf{2 0 0 8}$ & $\mathbf{2 0 0 9}$ & $\mathbf{2 0 1 0}$ & $\mathbf{2 0 1 1}$ & $\mathbf{2 0 1 2}$ & $\mathbf{2 0 1 3}$ & $\mathbf{2 0 1 4}$ \\
\hline Tipo de Cambio & 2,078 & 1,966 & 2,156 & 1,898 & 1,848 & 1,798 & 1,869 & 2,001 \\
Resultado del Ejercicio & $1,663,073$ & $2,114,649$ & $2,035,167$ & $2,539,356$ & $3,141,858$ & $3,648,900$ & $3,473,589$ & $3,962,481$ \\
Provisiones & $2,387,951$ & $3,658,222$ & $3,810,548$ & $4,198,105$ & $4,442,802$ & $5,540,276$ & $6,067,065$ & $5,830,276$ \\
Depreciaciones y Amortizaciones & 443,690 & 492,863 & 488,860 & 618,189 & 747,304 & 876,416 & $1,002,200$ & $1,223,161$ \\
NOPAT & $4,494,714$ & $6,265,734$ & $6,334,575$ & $7,355,650$ & $8,331,964$ & $10,065,593$ & $10,542,854$ & $11,015,918$ \\
Patrimonio Inicial & $6,496,095$ & $8,766,928$ & $10,776,341$ & $12,244,818$ & $16,588,547$ & $21,376,240$ & $26,147,284$ & $29,136,981$ \\
Retorno sobre Patrimonio & $69.19 \%$ & $71.47 \%$ & $58.78 \%$ & $60.07 \%$ & $50.23 \%$ & $47.09 \%$ & $40.32 \%$ & $37.81 \%$ \\
Costo de Capital & $6.82 \%$ & $8.90 \%$ & $7.50 \%$ & $8.00 \%$ & $9.00 \%$ & $8.80 \%$ & $8.30 \%$ & $8.60 \%$ \\
MARGEN (Retorno-Costo) & $62.37 \%$ & $62.57 \%$ & $51.28 \%$ & $52.07 \%$ & $41.23 \%$ & $38.29 \%$ & $32.02 \%$ & $29.21 \%$ \\
EVA & $4,051,680$ & $5,485,478$ & $5,526,350$ & $6,376,065$ & $6,838,995$ & $8,184,483$ & $8,372,629$ & $8,510,138$ \\
\hline
\end{tabular}

En el caso de Ecuador, el Retorno sobre Patrimonio inicial tiene un resultado del $42 \%$ en los años 2007 y 2008, que se reduce en el período 20092014 con un valor promedio de $31 \%$. Los datos se observan en la tabla 10.
(2007-2008) se observa una tendencia a incrementar, hasta el año 2009 en que los retornos se reducen, conformando una senda de menores retornos, salvo en el caso de Bolivia que se recupera en el año 2014.

\section{TABLA 10. CÁLCULO DEL EVA DE BANCA DE ECUADOR 2007-2014}

\begin{tabular}{lrrrrrrrr} 
AÑoS & $\mathbf{2 0 0 7}$ & $\mathbf{2 0 0 8}$ & $\mathbf{2 0 0 9}$ & $\mathbf{2 0 1 0}$ & $\mathbf{2 0 1 1}$ & $\mathbf{2 0 1 2}$ & $\mathbf{2 0 1 3}$ & $\mathbf{2 0 1 4}$ \\
\hline Resultado del Ejercicio & 253,942 & 282,223 & 217,611 & 260,902 & 394,848 & 314,270 & 268,041 & 334,702 \\
Provisiones & 182,410 & 241,605 & 223,262 & 216,216 & 319,125 & 449,491 & 351,126 & 365,650 \\
Depreciaciones y Amortizaciones & 90,108 & 90,667 & 85,132 & 86,934 & 91,217 & 106,758 & 112,121 & 124,708 \\
NOPAT & 526,460 & 614,495 & 526,006 & 564,051 & 805,189 & 870,519 & 731,288 & 825,060 \\
Patrimonio Inicial & $1,235,739$ & $1,437,891$ & $1,698,722$ & $1,869,668$ & $2,084,142$ & $2,482,836$ & $2,771,777$ & $2,909,115$ \\
Retorno sobre Patrimonio & $42.60 \%$ & $42.74 \%$ & $30.96 \%$ & $30.17 \%$ & $38.63 \%$ & $35.06 \%$ & $26.38 \%$ & $28.36 \%$ \\
Costo de Capital & $11.54 \%$ & $18.50 \%$ & $19.50 \%$ & $20.00 \%$ & $18.75 \%$ & $16.30 \%$ & $16.25 \%$ & $15.50 \%$ \\
MARGEN (Retorno - Costo) & $31.06 \%$ & $24.24 \%$ & $11.46 \%$ & $10.17 \%$ & $19.88 \%$ & $18.76 \%$ & $10.13 \%$ & $12.86 \%$ \\
EVA & 383,855 & 348,485 & 194,755 & 190,118 & 414,413 & 465,817 & 280,875 & 374,148 \\
\hline
\end{tabular}

En el caso de Perú, también es evidente que el Retorno sobre Patrimonio inicial se reduce posterior al año 2008, experimentando reducciones sucesivas. El promedio en el período 2007 al 2008 es de $55 \%$, y en el período 2009-2014 es de $44 \%$. El detalle de los cálculos se observa en la tabla 11.

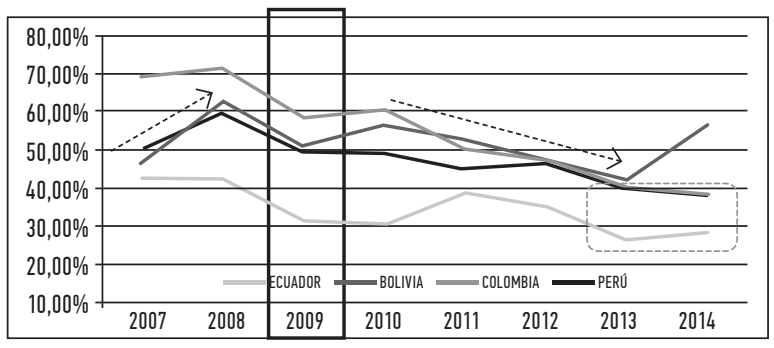

FIGURA 2: Evolución Retorno \% (EVA) - NOPAT / Patrimonio

TABLA 11. CÁLCULO DEL EVA DE BANCA DE PERÚ 2007-2014

\begin{tabular}{|c|c|c|c|c|c|c|c|c|}
\hline Años & 2007 & 2008 & 2009 & 2010 & 2011 & 2012 & 2013 & 2014 \\
\hline Tipo de Cambio & 3.124 & 2.941 & 3.006 & 2.825 & 2.752 & 2.634 & 2.721 & 2.846 \\
\hline Resultado del Ejercicio & 759,429 & $1,126,568$ & $1,077,857$ & $1,342,940$ & $1,565,804$ & $1,751,896$ & $1,825,139$ & $1,870,743$ \\
\hline Provisiones & 304,597 & 507,688 & 737,865 & 751,787 & 863,302 & $1,213,749$ & $1,273,798$ & $1,360,685$ \\
\hline Depreciaciones y Amortizaciones & 171,412 & 191,872 & 226,208 & 312,280 & 291,861 & 318,628 & 264,244 & 331,030 \\
\hline NOPAT & $1,235,438$ & $1,826,128$ & $2,041,930$ & $2,407,007$ & $2,720,967$ & $3,284,273$ & $3,363,181$ & $3,562,459$ \\
\hline Patrimonio Inicial & $2,463,197$ & $3,045,323$ & $4,148,288$ & $4,893,365$ & $6,078,317$ & $7,069,492$ & $8,473,028$ & $9,332,688$ \\
\hline Retorno sobre Patrimonio & $50.16 \%$ & $59.97 \%$ & $49.22 \%$ & $49.19 \%$ & $44.77 \%$ & $46.46 \%$ & $39.69 \%$ & $38.17 \%$ \\
\hline Costo de Capital & $6.82 \%$ & $8.90 \%$ & $7.50 \%$ & $8.00 \%$ & $9.00 \%$ & $8.43 \%$ & $7.85 \%$ & $7.55 \%$ \\
\hline MARGEN (Retorno-Costo) & $43.34 \%$ & $51.07 \%$ & $41.72 \%$ & $41.19 \%$ & $35.77 \%$ & $38.03 \%$ & $31.84 \%$ & $30.62 \%$ \\
\hline EVA & $1,067,448$ & $1,555,094$ & $1,730,808$ & $2,015,538$ & $2,173,918$ & $2,688,314$ & $2,698,048$ & $2,857,841$ \\
\hline
\end{tabular}




\section{DISCUSIÓN DE RESULTADOS}

El análisis de la creación de valor económico de los sistemas bancarios de la Comunidad Andina se puede resumir de la siguiente forma:

- Bolivia: en el sistema bancario el efecto de la crisis financiera mundial se puede explicar por un impacto en el crecimiento de los activos, se observa una reducción en el crecimiento en el 2009 y 2010 ubicándose en $18 \%$ y $10 \%$ respectivamente, para posteriormente presentar una recuperación con un promedio del $22 \%$. No existe un efecto negativo en el número de instituciones. La calidad de los activos medida por la proporción de activos productivos también se vio afectada, dado que del $82 \%$ en el período 2007-2008 descendió al 77\% en el período 2009-2012. Posteriormente se recupera a niveles antes de la crisis. La mezcla de activos en riesgo se vio afectada dado que sus resultados cambiaron de un $68 \%$ en el 2007, al 61\% en los años 2008 - 2009, para finalmente incrementar al $74 \%$ promedio para los años 2010 - 2014.

En el aspecto de rendimientos de activos y pasivos se observan períodos en los cuales los resultados cambian; para el período 2007-2009 existen indicadores superiores al período 2010-2013, que posteriormente se recuperaron en el 2014. La reducción en el rendimiento de los activos se compensa con la reducción de los pasivos con costo, lo cual equilibra el margen financiero, y permite que este se incremente en los períodos señalados, tal como se observa en la tabla 12.

TABLA 12. EVOLUCIÓN DE LOS RENDIMIENTOS DE LA BANCA DE BOLIVIA

\begin{tabular}{|lrrr}
\hline INDICADOR & $\mathbf{2 0 0 7 - 2 0 0 9}$ & $\mathbf{2 0 1 0 - 2 0 1 3}$ & $\mathbf{2 0 1 4}$ \\
\hline (+) Rendimiento de activos productivos & $8.77 \%$ & $7.60 \%$ & $8.89 \%$ \\
\hline Rendimiento de cartera & $10.88 \%$ & $9.53 \%$ & $10.84 \%$ \\
\hline Rendimiento de inversiones & $5.21 \%$ & $2.06 \%$ & $3.38 \%$ \\
\hline (-) Rendimiento de pasivos con costo & $4.10 \%$ & $2.02 \%$ & $2.45 \%$ \\
\hline (=) Margen Financiero & $4.67 \%$ & $5.57 \%$ & $6.44 \%$ \\
\hline
\end{tabular}

Cabe agregar que las provisiones aumentan de $1.61 \%$ en el 2007 , a $2.47 \%$ en el promedio de los años 2008-2010, reduciéndose al $1.82 \%$ promedio para los años 2011-2014.La relación de gastos operativos sobre activos presentó una reducción en el año 2009 en la relación ubicándose en el $3.85 \%$ y con una ligera tendencia creciente en los años siguientes.
- Colombia: el número de bancos no se redujo en el período de análisis. En el crecimiento de los activos después de la crisis que inicia en el 2008, año en el que presenta un resultado de $23.14 \%$, en el 2009 se observa una reducción de los activos en un $0.98 \%$, en los años siguientes se recuperaron. La calidad de los activos (proporción de activos productivos) presenta un resultado de $83 \%$ en el período 2007-2009, para posteriormente incrementarse al 96\% promedio en el período 2010-2014. La mezcla de activos en riesgo tuvieron un resultado promedio de $75 \%$ en los años 2007-2009, y se redujo a 67\% para los años 2010-2014. La mezcla de fuentes con costo presenta variaciones no significativas durante ese período. Esto se puede observar en la tabla 13.

\begin{tabular}{|c|c|c|}
\hline INDICADOR & 2007-2009 & $2010-2014$ \\
\hline (+) Rendimiento de activos productivos & $11.35 \%$ & $7.45 \%$ \\
\hline Rendimiento de cartera & $13.10 \%$ & $10.06 \%$ \\
\hline Rendimiento de inversiones & $6.06 \%$ & $2.09 \%$ \\
\hline (-) Rendimiento de pasivos con costo & $5 \%$ & $3.06 \%$ \\
\hline (=) Margen Financiero & $6.35 \%$ & $4.39 \%$ \\
\hline
\end{tabular}

Como complemento a estas cifras, se destaca que la relación de gastos operativos sobre activos promedio de $5 \%$ en el período 2007-2009 a un promedio de 4\% en el período 2010-2014. La relación comisiones e ingresos por servicios sobre el total de activos promedio de este indicador en el período 2007 - 2009 es de $4 \%$, que se ve reducido para el período 2010 - 2014 dado que presentó un promedio de $2.8 \%$.

- Ecuador: La crisis no tuvo efecto en el número de instituciones bancarias dado que la tendencia en el número de bancos fue a mantener o a incrementar hasta el año 2012; posterior a eso se reducen. El crecimiento de los activos presenta una desaceleración en el crecimiento posterior a la crisis, en el año 2009, en los años siguientes se recupera. La proporción de activos productivos se mantiene constante. La mezcla de activos en riesgo se reduce en el año 2009 a 60\% cuando el promedio del período 2007-2014 fue $63 \%$. La mezcla de fuentes con costo no presenta una variación significativa. En 
el indicador rendimiento de los activos productivos se ve un descenso en un punto porcentual en los años siguientes al 2008 , pasó de $8.63 \%$ a $7.62 \%$. La reducción en el margen financiero no es significativa, dado que se contrarresta con la reducción de los pasivos con costo. Esto se puede observar en la tabla 14.

TABLA 14. EVOLUCIÓN DE LOS RENDIMIENTOS DE LA BANCA DE ECUADOR

\begin{tabular}{|llll}
\hline INDICADOR & $\mathbf{2 0 0 7 - 2 0 0 8}$ & $\mathbf{2 0 0 9 - 2 0 1 1}$ & $\mathbf{2 0 1 2 - 2 0 1 4}$ \\
\hline (+) Rendimiento de activos productivos & $8.63 \%$ & $7.62 \%$ & $8.08 \%$ \\
\hline Rendimiento de cartera & $11.14 \%$ & $11.57 \%$ & $11.55 \%$ \\
\hline Rendimiento de inversiones & $4.09 \%$ & $1.32 \%$ & $1.67 \%$ \\
(-) Rendimiento de pasivos con costo & $3.80 \%$ & $2.85 \%$ & $2.86 \%$ \\
(=) Margen Financiero & $4.82 \%$ & $4.77 \%$ & $5.23 \%$ \\
\hline
\end{tabular}

Adicionalmente, en la relación de gastos / activos se observa una tendencia decreciente en todo el período analizado. En el indicador comisiones e ingresos por servicios / activos se evidencia una reducción que se da a partir del año 2008.

- Perú: El número de bancos no se afectó dado que no se observa una incidencia directa sobre ellos. El crecimiento de los activos se ve impactado por la crisis financiera que se refleja en la reducción del 4\% en el año 2009. La proporción de activos productivos se mantiene con pocas oscilaciones, salvo en el año 2009, en que incrementa $90.21 \%$ comparado con el promedio $2007-2014$ de $85.14 \%$. La mezcla de activos de riesgo, es decir la proporción de la cartera en los activos productivos, es de $66.91 \%$, menor al promedio $2007-2014$ de $70.71 \%$. No se evidencia un impacto en el indicador mezcla de fuentes con costo.

TABLA 15. EVOLUCIÓN DE LOS RENDIMIENTOS DE LA BANCA DE PERÚ

\begin{tabular}{lrr}
\hline INDICADOR & 2007-2008 & 2009-2014 \\
\hline (+) Rendimiento de activos productivos & $11.46 \%$ & $9.74 \%$ \\
\hline Rendimiento de cartera & $11.50 \%$ & $11.27 \%$ \\
\hline Rendimiento de inversiones & $11.37 \%$ & $6.18 \%$ \\
(-) Rendimiento de pasivos con costo & $3.45 \%$ & $2.38 \%$ \\
(=) Margen Financiero & $8.02 \%$ & $7.36 \%$ \\
\hline
\end{tabular}

CONCLUSIONES

Las conclusiones sobre los impactos de la crisis financiera mundial en la banca de la Comunidad Andina son:

1. En el año 2009 se observó una desaceleración en el crecimiento de la economía de los países andinos, dado que pasó de un promedio de variación del PIB de los cuatro países en el 2008 , de $6.3 \%$ a un $1.66 \%$ promedio en el año 2009.

2. El efecto de la crisis financiera mundial en los sistemas bancarios de los países de la Región Andina se refleja principalmente en una desaceleración del crecimiento de los activos, una disminución en el rendimiento de los activos productivos y del rendimiento de la cartera.

3. El total de los activos bancarios de la Comunidad Andina experimentó una reducción en el año 2009 de $-0.3 \%$, considerando que el crecimiento de los activos bancarios en el 2008 fue de $29 \%$ y se recuperó en los años siguientes en el período 2010-2012.

4. La crisis financiera mundial del 2008 impactó en la capacidad de creación de valor económico de la banca de la Comunidad Andina, lo que se refleja en el indicador Retorno sobre el patrimonio inicial, el cual se reduce posterior al año 2008 en la banca de todos los países.

5. Posterior al año 2009, el Retorno de la Banca medido por la división de las Utilidades operativas netas después de impuestos (NOPAT) para el Patrimonio Inicial, presenta una tendencia decreciente, pasando de un $60 \%$ promedio de los 4 países andinos en el 2008, a un 40\% en el año 2014.

6. El costo de capital o patrimonio promedio en el período 2007-2014 fue variable en cada uno de los países, de un $8.01 \%$ de Perú y $8.24 \%$ de Colombia, a un valor no tan distante de Bolivia en $11.66 \%$, y un porcentaje superior de costo de capital de $17 \%$ en Ecuador. Se afirma que el costo de capital tiene una incidencia relevante en el proceso de creación de valor económico, convirtiéndose en la variable que tiene más fuerza en la reducción del valor creado, dado que un incremento en el indicador costo de capital reduce el valor económico agregado.

7. La diferencia del Retorno según el método EVA y el Costo de patrimonio para la banca de cada país de la Región Andina nos permite identificar con mayor capacidad de creación de valor económico, siendo la banca de Colombia la de mayor Margen\% (Retorno - Costo) promedio en el período 2007-2014 resultando un 46\%, seguido por Bolivia y Perú con $40 \%$ y 39\% 
respectivamente, y finalmente $17 \%$ de Ecuador.

A partir de las conclusiones y hallazgos de esta investigación se definen futuras líneas de investigación que ameritan ser abordadas en próximas investigaciones, como son:

- Evaluación de la posición financiera de cada uno de los bancos de la Región Andina ante un incremento de la ex$^{-}$ posición de riesgo sistémico mediante pruebas de estrés bancario.

- Análisis del impacto en el sistema financiero del aumento excesivo del apalancamiento empresarial y un incremento de la probabilidad de quiebra derivado de un mayor endeudamiento.

- Análisis de las condiciones económicas y políticas que inciden en el Costo de patrimonio de la banca de cada país, y que producen asimetrías en el análisis del rendimiento bancario entre países.

\section{REFERENCIAS BIBLIOGRÁFICAS}

Allen, F., Myers, S.C., y Brealey, R.A. (2010). Principios de Finanzas Corporativas (Novena Edición ed.). México: McCraw-Hill Interamericana Editores S.A.

Amat, O. (1999). EVA Valor Económico Agregado - Un nuevo enfoque para optimizar la gestión empresarial, motivar a los empleados y crear valor.Barcelona: $\mathrm{Cru}^{-}$ po Editorial Norma.

Autoridad de Supervisión del Sistema Financiero de Bolivia (2015). Estadísticas de Intermediación Financiera [Archivo de datos]. Recuperado de https://www.asfi.gob.bo/Estadísticas/ IntermediaciónFinanciera/Boletines.aspx

Banco Mundial (2015). Indicadores de economía y crecimiento [Archivo de datos]. Recuperado de http://datos.bancomundial.org/indicador

Banco Mundial. (2014). Perspectivas económicas mundiales. Recuperado el 11 de Junio de 2014, de http://www.worldbank.org/content/dam/ Worldbank/GEP/GEP2014b/RegionalOverview_LAC_GEP_Jun2014_Sp.pdf

Barltrop, C. J., \& McNaughton, D. (1992). Banking Institutions in Developing Markets - Interpreting Financial Statements.Washington: The World Bank.

Biddle, G., Bowen, R. y Wallace, J. (1997). Does EVA beat earnings? Evidence on associations with stock returns and firm values. Journal of Accounting and Economics (24) 331 -336.

Costa, O. (2012). A framework for implementing EVA in Brazilian Banks. Journal of Money, Investment and Banking, 23.
Damodaran, A. (2014). Country Default Spreads and Risk Premiums [Archivo de datos]. Recuperado el 11 de Enero de 2015, de http://pages.stern. nyu.edu/ adamodar/

Fernández, P. (2013). Company valuation methods. Recuperado el 25 de Diciembre de 2014, de http:// papers.ssrn.com/sol3/papers.cfm?abstract id $=274973$

Fernández, P., \&Bermejo, V. (2008). ShareholderValue Creation of Banks in Spain: 1991-2007.Recuperado el 4 de Julio de 2012, de Social Science Research Network: http://papers.ssrn.com/sol3/papers. cfm?abstract_id=1092395

Fernández, P., Aguirreamalloa, J., \& Corres, L. (2011). Bancos españoles en 1991 - 2010 Creación de valor y rentabilidad para los accionistas. Recuperado el 2 de Julio de 2012, de Asociación Española de Banca: http://www.aebanca.es/internet/ groups/public/documents/publicaciones/00-201100441.pdf

Ferrari, C. (2008). Tiempos de Incertidumbre. Causas y consecuencias de la crisis mundial. Revista de Economía Institucional, 10(19), 55-78.

Fiordelisi, F., \& Molyneux, P. (2010). The determinants of shareholder value in European banking. Journal of Banking \& Finance, 34(6), 1189-1200.

Fraker, G. T. (2006). Using Economic Value Added (EVA) to Measure and Improve Bank Performance. Arizona, Estados Unidos.

Fondo Monetario Internacional. (2014). Informesobre la Estabilidad Financiera Mundial. Recuperado el 11 de Junio de 2014, de http://www.imf.org/external/spanish/pubs/ft/gfsr/2014/01/pdf/sums.pdf

Fondo Monetario Internacional. (2014). La solidez del sistema financiero. Recuperado el 11 de Junio de 2014, de http://www.imf.org/external/np/ exr/facts/spa/pdf/bankings.pdf

Gross, S. (2006). Banks and shareholdervalue-Anoverview of banks valuation and empirical evidence on shareholder value for banks. Deutscher Universitats-Verlag.

Hernández, R., Fernández-Collado, C., \& Baptista, P. (2007). Fundamentos de metodología de investigación. Madrid: McGraw - Hill Interamericana de España SA

Iñiguez Sánchez, R., Poveda Fuentes, F. (2001). Medidas de Creación de Valor para los accionistas: EVA vs. Beneficios. Revista Española de Financiación y Contabilidad (107), $207-237$.

Jácome Ortega, X. O. (2015). Método de análisis y supervisión a la gestión bancaria: $\mathrm{Mo}^{-}$ nitor de Banca de Ecuador (Tesis doctoral, Universidad Antonio de Nebrija, Madrid, España). Recuperado de https://www.researchgate.net/publication/287391700_Metodo_ 
de_analisis_y_supervision_a_la_gestion_bancaria_Monitor_de_Banca_de_Ecuador.

Koller, T., Goedhart, M., \& Wessels, D. (2010). Valuation: Measuring and Managing the Value of Companies. New Jersey: John Wiley and Sons, Inc.

López Lubián, F., \& Vilela, E. (2000). Creación de Valor en Entidades Bancarias. Harvard Deusto Business Review.

Neira Orjuela, F. (2009). Migración, remesas e indicadores económicos en la Comunidad Andina. Latinoamérica, Revista de Estudios Latinoamericanos(49), 79-96.

Ocampo, J. A. (2009). Impactos de la crisis financiera mundial sobre América Latina. Revista CEPAL(97), 9-32.

Ocampo, J. A. (2011). ¿Cómo fue el desempeño de América Latina durante la crisis financiera global? Ensayos Económicos, 7-33.

Popa, G., Mihailescu, L., \& Caragea, C. (2009). EVA - Advanced method for performance evaluation in banks. Economia seria Management, 12(1).

Serrano Rodríguez, J. (2009). Crisis financiera de 2008: la visión un año después. Revista Soluciones de Postgrado EIA(4), 77-105.

Stern, J. M., Shiely, J. S., \& Ross, I. (2002). El desafío del EVA: Cómo implementar el cambio del valor agregado en la organización. Bogotá: Editorial Norma S.A.

Stewart, B. (1991). The Quest for Value: The EVA Management Guide.Estados Unidos: Harper Collins, Publishers Inc.

Superintendencia de Banca, Seguros y AFP de Perú. (2015). Información Estadística de Banca Múl- tiple [Archivo de datos]. Recuperado de http:// www.sbs.gob.pe/app/stats/EstadisticaBoletinEstadistico.asp?p=1

Superintendencia de Bancos y Seguros del Ecuador. (2015). Información Financiera y Datos Estadísticos [Archivo de datos]. Recuperado de http://www.sbs.gob.ec/practg/sbs_index?vp_ art_id=29\&vp_tip=2\&vp_buscr $=41$

Superintendencia Financiera de Colombia. (2015). Informes y cifras de establecimientos de créditos [Archivo de datos]. Recuperado de https:// www.superfinanciera.gov.co/jsp/loader.jsf?ls ervicio $=$ Publicaciones\&lTipo $=$ publicaciones\& 1 Funcion=loadContenidoPublicacion\&id $=60775$

Venanzi, D. (2010). Financial performance measures and value creation: a review. Recuperado el 22 de Diciembre de 2014, de http://papers.ssrn.com/ sol3/papers.cfm?abstract_id=1716209

Wittrup, K., \& Jensen, J. A. (2012). Shareholder Value in Banks (Tesis de Maestría, Universidad Aarhus). Obtenido de http://pure.au.dk/portal-asb-student/ files/47810473/Shareholder_Value_in_Banks_ Thesis.pdf

Worthington , A., \& West, T. (2001). Economic Value-Added: A Review of the Theoretical and Empirical Literature. Asian Review of Accounting, 9(1), 67-86.

Zurita González, J., Martínez Pérez, J., \& Rodríguez Montoya, F. (2009). La crisis financiera y económica del 2008. Origen y consecuencias en los Estados Unidos y México. Recuperado el 10 de Diciembre de 2015, de http://elcotidianoenlinea.com. $\mathrm{mx} / \mathrm{pdf} / 15703 . p d f$ 